\title{
Cognitive Reserve Profiles in Chronic Schizophrenia: Effects on Theory of Mind Performance and Improvement after Training
}

\author{
Mariachiara Buonocore, ${ }^{1}$ Margherita Bechi, ${ }^{1}$ Paola Uberti, ${ }^{2}$ Marco Spangaro, ${ }^{1,2}$ Federica Cocchi, ${ }^{1}$ Carmelo Guglielmino, ${ }^{1}$ \\ Laura Bianchi, ${ }^{1}$ Antonella Rita Mastromatteo, ${ }^{1,2}$ Marta Bosia, ${ }^{1,2}$ AND Roberto Cavallaro ${ }^{1,2}$ \\ ${ }^{1}$ Department of Clinical Neurosciences, IRCCS San Raffaele Scientific Institute, Milano, Italy \\ ${ }^{2}$ Università Vita-Salute San Raffaele, Milano, Italy \\ (Received February 17, 2017; Final Revision September 14, 2017; Accepted December 19, 2017; First Published OnLine March 20, 2018)
}

\begin{abstract}
Objectives: Cognitive reserve (CR), defined as individual differences in the ability to cope with brain damage, seem to be associated to the several psychopathological features in psychiatric patients, such as the functional outcome. This study aims to identify different profiles of CR by combining intelligence quotient (IQ) and premorbid functioning, two measures independently associated to CR in previous works, as well as to explore CR effect on both Theory of Mind (ToM) baseline performance and improvement after socio-cognitive trainings. Methods: Sixty patients with chronic schizophrenia underwent a socio-cognitive rehabilitation. All patients were assessed for psychopathology, neurocognition, and ToM at baseline and post-treatment. CR profiles were explored with K-means cluster analysis, while differences between clusters in both baseline assessments and post-treatment ToM improvement, were analyzed by means of analysis of variance and repeated measures analysis of covariance. Results: The analysis revealed three CR profiles, respectively, characterized by low early premorbid functioning and mild intellectual impairment, average/high early premorbid functioning trend with moderate intellectual impairment and good early premorbid functioning associated to IQ within normal limits. Analyses showed a significant effect of CR on both baseline ToM performance and treatment outcome: patients with higher CR reached significantly better ToM scores. Conclusions: These results underline the clinical relevance of defining CR profiles of patients to customize trainings: subjects with a lower CR may benefit from more intensive programs. A deeper knowledge about CR may considerably increase our understanding of individual differences and thus potentiate treatment outcome. (JINS, 2018, 24, 563-571)
\end{abstract}

Keywords: Psychosis, Cognition, Social cognition, Intelligence quotient, Rehabilitation, Premorbid functioning

\section{INTRODUCTION}

Cognitive reserve (CR), defined as "individual differences in how people process tasks which allow some to cope better than others with brain pathology" (Stern, 2002), has been proposed as a crucial etiologic factor in the development and severity of neuropsychiatric disorders (Anaya et al., 2016; Barnett, Salmond, Jones, \& Sahakian, 2006; Forcada et al., 2015; O'Shea et al., 2015). In schizophrenia, evidence suggests an effect of CR on both onset and prognosis (Barnett et al., 2006; Evans et al., 2004; McGurk et al., 2000; Velligan et al., 1997), as well as on the integrity and development of the brain (Amoretti et al., 2016; Levitt, O’Donnell, McCarley, Nestor, \& Shenton, 1996; Neumann, Baum, Walker, \& Lewine,

Correspondence and reprint requests to: Marta Bosia, Department of Clinical Neurosciences, IRCCS San Raffaele Scientific Institute, Via Stamira d'Ancona 20, 20127 Milano, Italy. E-mail: bosia.marta@hsr.it
1996). The practical importance of using multiple measures of $\mathrm{CR}$ to achieve a more comprehensive understanding of its association with cognition has been highlighted (Anaya et al., 2016; Stern, 2003).

So far, premorbid functioning and IQ appear the most reliable measures of $\mathrm{CR}$ in schizophrenia (Amoretti et al., 2016; Cole, Apud, Weinberger, \& Dickinson, 2012; Cuesta et al., 2015; Leeson et al., 2011). Leeson and colleagues (Leeson et al., 2011) identified three possible "IQ trajectories", based on evaluation of premorbid and current IQ, respectively, characterized by stable average/high range IQ, intellectual deterioration and stable low IQ. Examining the relationship between these three IQ trajectories and psychopathological, cognitive and functional measures through a longitudinal study, the authors found that both stable low IQ and intellectual deterioration were associated with cognitive deficits and more prominent symptoms at onset and with worse occupational outcome after 3 years. 
On the other hand, Cole et al. (2012) focused on premorbid adjustment, still identifying three different profiles of premorbid functioning. The first group showed consistently adequate-to-good social and academic premorbid functioning, the second showed initially good functioning and deterioration with time until onset, the third showed poor functioning in childhood that declined further during the years up to diagnosis. Significant differences were reported among these three groups, the first showing better functional and cognitive outcome.

In general, the previous data support the role of both IQ and premorbid functioning as reliable measures of CR and highlight the impact of $\mathrm{CR}$ on functional and cognitive outcome in schizophrenia. Still, it is to notice that measures of IQ as indicator of CR vary across studies, including both premorbid IQ, as well as estimates based on current IQ evaluations (Ferreira et al., 2016; Kim et al., 2015; Nucci et al., 2011). In this view, an interesting key issue is to analyze the so far less explored relation between CR and socio-cognitive domains. Theory of Mind (ToM), defined as the ability to reflect upon one's own and other persons' mental states including desires, beliefs, knowledge, intentions, and feelings (Frith, 1993; Frith \& Frith, 2003), appears of particular relevance as it predicts interpersonal functioning. For this reason, in the past decade several rehabilitation programs have been developed specifically to improve ToM performance in patients with schizophrenia (Bechi et al., 2015; Bechi et al., 2012, 2013; Combs, Tosheva, Wanner, \& Basso, 2006; Kurtz, Gagen, Rocha, Machado, \& Penn, 2016; Penn \& Combs, 2000). Although promising, results of these interventions are still heterogeneous, highlighting the need to further explore factors contributing to ToM performance and their role as putative predictors of ToM rehabilitation (Fliss et al., 2016).

Based on these premises, the present study will pursue the following aims: (1) to identify different clusters of CR in a sample of clinically stabilized patients with schizophrenia by combining measures of IQ and premorbid functioning; (2) to investigate the relationship between CR clusters and clinical, neurocognitive and ToM measures; (3) to analyze the effect of CR clusters on ToM improvement after a socio-cognitive rehabilitation program. We hypothesized that patients presenting higher CR could be buffered against the diseaseassociated deterioration and thus show better ToM performance and greater ToM enhancement after training.

\section{METHODS}

\section{Subjects}

Sixty outpatients were enrolled at IRCCS San Raffaele Scientific Institute, Milan, Italy. They all met DSM IV-R criteria for schizophrenia, as determined by trained psychiatrists by using medical records and DSM IV-R Structured Clinical Interview, and were clinically stabilized. Patients were treated with a stable dose of the same antipsychotic therapy for at least 3 months and remained on the same medication throughout the study. Exclusion criteria were: substance dependence or abuse, co-morbid diagnosis on Axis I or II, major neurological illnesses, perinatal trauma, and mental retardation. All subjects provided informed consent to a protocol approved by the local Ethical Committee, following the principles of the Declaration of Helsinki.

\section{Design}

All patients were assessed before starting sociocognitive rehabilitation programs and after the end of the treatment (T1).

All enrolled patients were assessed at baseline and after the end of an integrated treatment, which included a standard rehabilitation therapy (SRT) and a socio-cognitive rehabilitation program.

In details, all patients were treated with the following integrated treatment: (1) 3 months of one of both of the sociocognitive rehabilitation program [ToMI or Social Cognition and Interaction Training (SCIT)] that have been previously demonstrated to improve ToM and are described in detail in "Interventions" (Bechi et al., 2015, 2012, 2013). Our previous study have shown that there are no statistically significant differences between ToMI and social cognitive training (SCT) in improving ToM abilities and in Quality of life improvement (Bechi et al., 2015). (2) 6 months of SRT, performed including the integrated psychological therapy (Brenner, Hodel, Roder, \& Corrigan, 1992), social skills training programs for residential, vocational, recreational functioning (Roder et al., 2002), and psychoeducation.

All interventions were conducted by trained psychologists with at least 5 years of experience in cognitive behavioral therapy with patients affected by schizophrenia.

\section{Assessments}

All patients were assessed for psychopathology, premorbid adjustment, intellectual level, neurocognitive and ToM domains. ToM abilities were also re-evaluated after the rehabilitative interventions. All assessments were administered by trained psychologists, except for the psychopathologic evaluation, performed by trained psychiatrists.

\section{Psychopathological assessment}

Psychopathology was assessed by means of the Positive and Negative Syndrome Scale (PANSS) (Kay, Fiszbein, \& Opler, 1987), a standardized measurement for typological and dimensional symptoms. Positive, Negative, and General subscales and their sum (Total score) were used for analyses.

\section{Premorbid functioning assessment}

The level of functioning before onset was evaluated by means of the Premorbid Adjustment Scale (PAS) (Cannon-Spoor, Potkin, \& Wyatt, 1982), a retrospective interview mainly 
focused on an individual's social and academic adjustment before illness onset, administered to the patients and their parents. It covers childhood (up to age 11), early adolescence (12 to 15), late adolescence (17 to 18), and adulthood (19 and older). According to scale instructions, adulthood was not assessed in patients with onset before or at 19 years of age. To maintain adequate groups size and to enhance generalizability, we excluded PAS adulthood scores from data analysis. A general score was also included, assessing the highest level achieved before onset with respect to education, school or job function, and quality of life.

Ratings are made on a 0 to 6 Likert scale, with 0 indicating perfect adjustment and 6 indicating severe impairment. Only those life periods that are premorbid by definition are rated regardless of the present age of the subject. The total score for each life period is calculated by summing up all subscale scores and dividing by the total possible score. An overall score can be calculated by averaging the total scores of all time-periods.

\section{Intelligence quotient assessment}

Intellectual functioning was assessed by means of the Wechsler Adult Intelligence Scale - Revised, Italian Version (WAIS; Wechsler, 1997). Verbal, Performance, and Total IQ scores were used for the analyses. For the measurement of IQ, raw scores were all age-adjusted.

\section{Neuropsychological assessment}

Neurocognitive domains were evaluated with the Italian version of the Brief Assessment of Cognition in Schizophrenia (BACS) (Anselmetti et al., 2008; Keefe et al., 2004), including the following tasks: word recall (verbal memory), digit sequencing (working memory), token motor task (psychomotor speed and coordination), symbol coding (processing speed), semantic and phonemic fluency (verbal fluency), and Tower of London (executive functionsplanning).

Analyses were performed on raw scores for BACS subtests.

\section{Theory of Mind assessment}

ToM was assessed using the ToM Picture Sequencing Task (PST) (Brüne, 2003b), consisting of six cartoon picture stories depicting (1) two scenarios where two characters cooperate, (2) two scenarios where one character deceives a second character, and (3) two scenarios with two characters cooperating to deceive a third. In the Sequencing task, measure of non-verbal and affective ToM processing, four cards were presented face-down in mixed order, the participants were asked to turn the cards over and to order them in a logical sequence of events, two points were given for the first and last correctly sequenced cards and one point each for correct sequencing of the two middle cards.

In addition, a ToM questionnaire with 23 questions was administered to the subjects to test their ability to appreciate the mental states of the characters involved in the cartoon stories, as a measure of "Cold ToM." The questions referred to the mental states of the characters according to different levels of complexity and included first to third false belief questions, questions involving the understanding of cheating detection and two reality questions, basically included to rule out major attentional problems. An answer was considered incorrect and scored 0 if involving errors about the facts depicted in the story or inappropriate inference on characters' mental states, motivations or beliefs (Brüne, 2003a).

The variables of interest of this study were: first, second, and third order false belief questions, Total Questionnaire score, as measures of "Cold ToM", Total Sequencing score, as measure of "Hot ToM" and PST Total score, as global measure of ToM abilities (Bechi et al., 2013b). As previously reported (Bechi et al., 2012), this scale shows a good internal consistency (Cronbach's $\alpha$ coefficient $=0.86$ ).

\section{Interventions}

The Theory of Mind Intervention (ToMI) was conducted by trained psychotherapists over five modules divided into 18 sessions (1-h session twice a week) on groups of about five members, using comic strips and cartoons depicting human social interactions. The modules were executed in increasing order of complexity, with the first three modules focusing on cognitive ToM and the last two on affective ToM. Assuming that ToM difficulties in schizophrenia are associated with an inability to extract relevant data from the context (Frith et al., 1996; Pickup \& Frith, 2001), we trained patients to recognize the relevant details, to collect every concrete and meaningful piece of information they saw (place, time, characters' actions and physical features), to read the verbal part of comic strips and to identify literal meaning. Then we asked patients to interpret hidden meanings using all the information collected and to further hypothesize interpretations, based on expressed emotions, relationships between characters, implicit motivations, and mental states. Answers were read aloud and then patients had to assign a title to the story. A guided discussion of each hypotheses followed (more details in Bechi et al., 2013).

The SCT was conducted by trained psychotherapists over 12 weeks (one 1-h sessions/week) on groups of about five members, using short videos depicting human social interactions, selected from international movies. Scenes lasted between $30 \mathrm{~s}$ and $70 \mathrm{~s}$ and suggest recognition of emotions and ToM abilities to be correctly comprehended. In each session, two or three clips were presented and could be viewed several times, according to the requests of the patients. A guided discussion of hypotheses followed (more details in Bechi et al., 2012).

The SRT was performed including the integrated psychological therapy (Brenner et al., 1992), social skills training programs for residential, vocational, recreational functioning (Roder et al., 2002), and psychoeducation. 


\section{Statistical Analyses}

The cluster analysis allows to categorize subjects into consistent groups determining clusters of participants displaying small within-cluster variation relative to the between-cluster variation (Dillon \& Goldstein, 1984). In particular, K-means cluster analysis is a non-hierarchical form of cluster analysis appropriate when you already have hypotheses (Lysaker et al., 2010). It is the most commonly used method in data for which the desired number of clusters $(\mathrm{K})$ in a sample is decided a priori (Bell, Johannesen, Greig, \& Wexler, 2010). As in previous studies (Bell et al., 2010; Lysaker et al., 2010), we chose this procedure with the aim to identify three meaningful patients' profiles, significantly different from one another, as putative indexes of CR.

We compared the adjustment of different clustering solutions with 1, 2, 3, 4, and 5 clusters using the Bayesian information criterion (BIC). The inspection of the BIC values showed a first maximum of 1683,562 for the 2-cluster solution and a second maximum BIC of 1694,179 for the threecluster solution. Given the very slight difference in BIC values, we opted for the three-cluster solution, as it fitted the data and it is in line with previous literature. Then, each subject was assigned to a most probable cluster (Cole et al., 2012; Gilbert et al., 2014; Leeson et al., 2011; Ochoa et al., 2013; Renwick et al., 2015).

Analysis of variance (ANOVA) and chi-square test (for dichotomic variables) were performed to compare the groups produced by the cluster analysis, on the basis of their IQ and PAS scores, on demographic, clinical, neurocognitive, and ToM basal measures. After Bonferroni's correction for multiple comparisons, the significance level was set at $p<.003$. Post hoc analyses with Fisher least significant difference (LSD) were used to yield significant differences between groups. For these analyses, we had a power of 0.80 to detect a medium-large effect size $(f=0.4)$ at a type 1 error level of $p<.05$ (Steiger, 2004).

To compare the effects of ToMI and SCT on ToM improvement, a one-way ANOVA was performed with ToM change (pre-treatment score subtracted from post-treatments) as dependent variable and group (ToMI $v s$. SCT) as independent variable.

A repeated measures ANCOVA was performed to analyze significant differences between clusters on the degree of ToM improvement after training. To take into account also other possible confounding factors independently contributing to
ToM performance, beyond the effect of the identified cluster groups, Pearson correlation analysis was performed between ToM performance and other cognitive functions, measured through BACS. Variables that resulted significantly correlated with ToM performance after Bonferroni correction were then entered as covariates in the ANCOVA. Fisher LSD post hoc test followed. For this analysis, we had a power of 0.93 to detect a medium effect size $(f=0.25)$.

G*Power Software version 3.1.7 was used for Power Analysis. All other analyses were performed with STATISTICA Software for Windows, version 8 (StatSoft Inc., Tulsa, OK).

\section{RESULTS}

The ANOVA showed no significant differences in ToM improvement between ToMI and SCT.

\section{Cluster Analysis}

The K-means cluster analysis produced three groups with 22, 20, 18 members each: Cluster $1(\downarrow P A S \pm I Q)$ shows low early premorbid functioning scores and mild intellectual impairment; cluster $2( \pm$ PAS $\downarrow$ IQ) shows an average/high early premorbid functioning trend, but moderate intellectual impairment; cluster 3 ( $\uparrow \mathrm{PAS} \uparrow \mathrm{IQ}$ ) shows both good early premorbid functioning and within normal limits IQ. Table 1 shows the mean PAS and WAIS-R scores for each of the three clusters.

Results of variance analysis of the generalized cluster analysis k-means showed significant effects for: Verbal IQ $(F=20.0 ; p \leq .001)$, Performance IQ $(F=10.22 ; p \leq .001)$, Total IQ $(F=18.93, \quad p \leq .001), \quad$ Childhood $(F=48.20$; $p \leq .001)$, Early adolescence $(F=92.12 ; p \leq .001)$, Later adolescence $(F=25.49 ; p \leq .001)$, General $(F=13.60 ; p<.001)$.

\section{Effect of Clusters on Baseline Assessments}

Table 2 shows demographic, clinical, neurocognitive and ToM features, stratified by cluster groups. No significant difference was found for any of the variables considered, except for ToM performance. Post hoc analysis showed that cluster 3 ( $\uparrow$ PAS $\uparrow$ IQ) displayed a significantly higher PST Total Score than both

Table 1. Premorbid adjustment scores at different time points and intellectual level stratified by clusters

\begin{tabular}{|c|c|c|c|c|c|c|c|}
\hline & Childhood & Early adolescence & Later adolescence & General & Verbal IQ & Performance IQ & Total IQ \\
\hline $\begin{array}{l}\text { Cluster } 1 \\
\qquad \begin{array}{l}\downarrow \mathrm{PAS} \pm \mathrm{QI} \\
N=22\end{array}\end{array}$ & $49.11 \pm 15.05$ & $57.72 \pm 10.45$ & $59.84 \pm 12.32$ & $55.13 \pm 10.14$ & $90.95 \pm 11.93$ & $83.68 \pm 12.21$ & $86.68 \pm 9.83$ \\
\hline $\begin{array}{l}\text { Cluster } 2 \pm \mathrm{PAS} \downarrow \mathrm{QI} \\
\quad N=20\end{array}$ & $27.29 \pm 8.05$ & $33.83 \pm 10.93$ & $38.14 \pm 16.65$ & $51.2 \pm 11.30$ & $79 \pm 5.88$ & $74.8 \pm 8.63$ & $76.8 \pm 5.12$ \\
\hline $\begin{array}{l}\text { Cluster } 3 \\
\quad \uparrow P A S \uparrow Q I \\
N=18\end{array}$ & $18.28 \pm 8.94$ & $25.58 \pm 9.22$ & $38.66 \pm 15.84$ & $40.74 \pm 8.7$ & $98.61 \pm 9.7$ & $90.94 \pm 11.63$ & $94.72 \pm 10.64$ \\
\hline
\end{tabular}


Table 2. Baseline demographic, clinical, neurocognitive, and ToM assessments stratified by clusters

\begin{tabular}{|c|c|c|c|c|c|c|}
\hline & Sample & $\begin{array}{c}\text { Cluster } 1 \\
\downarrow \mathrm{PAS} \pm \mathrm{IQ}\end{array}$ & Cluster $2 \pm$ PAS $\downarrow$ IQ & $\begin{array}{c}\text { Cluster } 3 \\
\uparrow \mathrm{PAS} \uparrow \mathrm{IQ}\end{array}$ & $\mathrm{Chi}^{2} / \mathrm{ANOVA}$ & \\
\hline & $n=60$ & $n=22$ & $n=20$ & $n=18$ & $\mathrm{X}$ & $p$-Value \\
\hline \multirow[t]{2}{*}{ Gender (M/F) } & $40 / 20$ & $12 / 10$ & $13 / 7$ & $15 / 3$ & 3.73 & .15 \\
\hline & Mean $\pm S D$ & Mean $\pm S D$ & Mean $\pm S D$ & Mean $\pm S D$ & $F$ & $p$-Value \\
\hline Age (yr) & $33.55 \pm 9.23$ & $34.85 \pm 9.37$ & $31.57 \pm 9.22$ & $34.11 \pm 9.25$ & 0.67 & .52 \\
\hline Education (yr) & $11.87 \pm 2.48$ & $12.52 \pm 2.69$ & $10.89 \pm 2.53$ & $12.16 \pm 1.91$ & 2.43 & .10 \\
\hline Age of onset & $24.37 \pm 6.37$ & $24.71 \pm 6.97$ & $23.94 \pm 6.68$ & $24.44 \pm 5.61$ & 0.07 & .93 \\
\hline Illness duration $(\mathrm{yr})$ & $9.17 \pm 6.91$ & $10.14 \pm 7$ & $7.63 \pm 5.42$ & $9.66 \pm 8.21$ & 0.72 & .49 \\
\hline Antipsychotic dose (Cpz-eq) & $243.6 \pm 67.9$ & $238,01 \pm 67,26$ & $283,27 \pm 61,40$ & $210 \pm 75,20$ & 0.30 & .74 \\
\hline \multicolumn{7}{|l|}{ PANSS } \\
\hline Positive subscale & $17.35 \pm 4.71$ & $17.30 \pm 4.43$ & $19.3 \pm 2.98$ & $15.73 \pm 5.83$ & 2.09 & .14 \\
\hline Negative subscale & $20.51 \pm 3.7$ & $19.11 \pm 3.91$ & $22.28 \pm 2.98$ & $21.38 \pm 3.3$ & 2.57 & .09 \\
\hline General subscale & $37.14 \pm 7.78$ & $36.05 \pm 7.34$ & $38 \pm 7.71$ & $37.86 \pm 8.73$ & 0.33 & .72 \\
\hline Total score & $76.37 \pm 13.77$ & $73.3 \pm 13.23$ & $83.23 \pm 8.78$ & $74.53 \pm 16.51$ & 2.37 & .10 \\
\hline \multicolumn{7}{|l|}{ BACS } \\
\hline Verbal memory & $38.73 \pm 9.52$ & $41.57 \pm 9.94$ & $34.81 \pm 9.97$ & $39.77 \pm 7.3$ & 2.77 & .07 \\
\hline Working memory & $16.65 \pm 3.4$ & $16.49 \pm 3.64$ & $15.55 \pm 3.12$ & $18.19 \pm 2.99$ & 2.68 & .08 \\
\hline Psychomotor speed and coordination & $65.71 \pm 18.04$ & $66.9 \pm 19.77$ & $63.89 \pm 14.88$ & $66.29 \pm 19.88$ & 0.15 & .86 \\
\hline Verbal fluency & $39.14 \pm 10.78$ & $40.85 \pm 11.51$ & $35.07 \pm 9.69$ & $41.43 \pm 10.32$ & 2.10 & .13 \\
\hline Processing speed & $37.72 \pm 9.4$ & $39.29 \pm 9.21$ & $34.05 \pm 8.51$ & $39.76 \pm 9.86$ & 2.26 & .11 \\
\hline Executive functioning & $14.09 \pm 3.46$ & $14.42 \pm 3.68$ & $13.1 \pm 3.71$ & $14.74 \pm 2.82$ & 1.19 & .31 \\
\hline \multicolumn{7}{|l|}{ PST } \\
\hline Total Score pre & $43.79 \pm 10.42$ & $44.66 \pm 10.69$ & $36.94 \pm 8.69$ & $50 \pm 7.46$ & 9.57 & $<.001$ \\
\hline Total Score post & $47.27 \pm 8.17$ & $50.18 \pm 5.81$ & $47.76 \pm 8.59$ & $44.75 \pm 8.77$ & 1.15 & .22 \\
\hline
\end{tabular}

clusters $1(\downarrow \mathrm{PAS} \pm \mathrm{IQ})$ and $2( \pm$ PAS $\downarrow$ IQ) with effect sizes of 0.5 and 1.5 , respectively (all $p \leq .0001$ ).

\section{Correlation Analysis}

Correlations between ToM performance and other cognitive functions are reported in Table 3 After Bonferroni's correction, a significant correlation was observed only for BACS subtest

Table 3. Correlations between neurocognitive and ToM performance at baseline (the reported $p$ values are adjusted for multiple comparisons according to Bonferroni's correction).

\begin{tabular}{ll}
\hline \hline & \multicolumn{1}{c}{ PST } \\
\hline BACS & \\
Verbal memory & $\mathrm{r}=0.22$ \\
& $p=.51$ \\
Working memory & $\mathrm{r}=0.33$ \\
& $p=.06$ \\
Psychomotor speed and coordination & $\mathrm{r}=0.29$ \\
& $p=.09$ \\
Verbal fluency & $\mathrm{r}=0.25$ \\
& $p=.25$ \\
Processing speed & $\mathrm{r}=0.28$ \\
& $p=.07$ \\
Executive functions & $\mathrm{r}=0.40$ \\
& $p=.0007 *$ \\
\hline \hline
\end{tabular}

Tower of London, evaluating executive functioning in the subcomponent of planning $(p=.0007 ; \mathrm{r}=0.40)$.

\section{Effect of clusters on ToM improvement}

Repeated Measures ANCOVA with pre and post-treatment ToM performance as dependent variable, cluster groups as categorical factor, Time as fixed factor, and Tower of London as covariate showed significant main effects and interactions, as reported below: Cluster: $F=5.36, \mathrm{df}=2, p=.007$; Tower of London: $F=9.36, \mathrm{df}=1, p=.003$; Time: $F=5.60, \mathrm{df}=1$, $p=.021$; Time*Tower of London: $F=1.57$, df $=1, p=.22$; Time*Cluster: $F=3.47$, df $=2, p=.038$.

Fisher post hoc showed several significant differences. In details, differences between clusters in baseline performance were confirmed, as reported in the previous paragraph. Moreover, we observed a significant improvement within clusters from baseline to after-treatment evaluation in cluster $1(\downarrow \mathrm{PAS} \pm$ IQ) $(p=.02)$ and $2( \pm$ PAS $\downarrow$ IQ) with effect sizes of 0.42 and 1.2, respectively (all $p<.0001$ ). Finally, post-treatment scores significantly differed only between cluster 3( $\uparrow$ PAS $\uparrow I Q)$ and 2 $( \pm$ PAS $\downarrow$ IQ) with an effect size of $0.7(p=.03)$, the latter showing worse performance.

\section{DISCUSSION}

To the best of our knowledge, this is the first study exploring CR profiles in a sample of clinically stabilized patients with 
chronic schizophrenia, through combined measures of IQ and premorbid functioning, as well as assessing the relationship between CR and both ToM performance and its improvement after socio-cognitive rehabilitation trainings.

The cluster analysis showed three relevant profiles of CR displaying small within-cluster variation with the maximum between-cluster variation, respectively, characterized by low early premorbid functioning scores and mild intellectual impairment $(\downarrow P A S \pm I Q)$, average/high early premorbid functioning trend with moderate intellectual impairment; $( \pm$ PAS $\downarrow$ IQ), and high early premorbid functioning trend with within normal limits IQ ( $\uparrow$ PAS $\uparrow I Q)$. This result substantially merges, through a more comprehensive assessment, previous works by Leeson et al. (2011) and Cole et al. (2012), which identified three paths of development, respectively, with premorbid adjustment and both premorbid and current IQ.

In particular, Leeson and colleagues (Leeson et al., 2011) found stable high functioning group, similar to the current cluster 3 ( $\uparrow$ PAS $\uparrow I Q$ ); a deteriorating group, that may show some similarities with cluster $2( \pm$ PAS $\downarrow$ IQ); a stable low functioning group similar to cluster $1(\downarrow \mathrm{PAS} \pm \mathrm{IQ})$ that shows low premorbid functioning scores and average IQ. The three profiles identified in this study further support Cole and Leeson hypothesis, providing additional evidence of the clinical significance of these trajectories, validating the value of premorbid functional adjustment and IQ as measures of CR in patients affected by schizophrenia (Levitt et al., 1996; Neumann et al., 1996).

With respect to the relationship between CR clusters and clinical, neurocognitive, and ToM baseline measures, the analysis showed significant differences between clusters only for ToM performance, while no significant effects were yielded for psychopathology, as assed by PANSS, nor neurocognition, evaluated with the BACS. These latter negative results are apparently in contrast with previous work suggesting that CR predicts symptoms severity (Leeson et al., 2011), as well as core cognitive domains, through the illness progression (de la Serna et al., 2013), and it also affects the capacity to improve in cognition after a cognitive remediation program (Kontis, Huddy, Reeder, Landau, \& Wykes, 2013). However, it is to notice that these studies involved a longitudinal evaluation of the clinical course, while in our, psychopathology and neurocognition were assessed only at baseline. Moreover, our classification of premorbid functioning did not clearly distinguished patients according to trajectories, but only to general level, limiting comparison with previous works.

Finally, with respect to symptoms dimension, we need to consider that all patients, in our work, were clinically stabilized and thus highly homogeneous in terms of psychopathology. This criterion allowed a deeper investigation of "core" cognitive and socio-cognitive abilities, as the target of this study, limiting the influence of acute phase symptomatology, however, it may prevent us from drawing conclusions on the relationship between CR and psychopathology.

Regarding ToM abilities, results instead suggested a significant influence of CR. In details, cluster 3 ( $\uparrow$ PAS $\uparrow$ IQ) showed significantly higher ToM performance, as compared to both clusters $1(\downarrow \mathrm{PAS} \pm \mathrm{IQ})$ and $2( \pm$ PAS $\downarrow \mathrm{IQ})$. The contribute of $\mathrm{CR}$ to socio-cognitive dimensions, core domains affected by schizophrenia, although still less explored, is supported by previous evidence, showing an association between ToM and both intellectual resources and premorbid functioning (Brüne, 2003b; Mazza, De Risio, Surian, Roncone, \& Casacchia, 2001). Moreover, given that $\mathrm{CR}$ is considered a protective factor against the global decline related to cerebral dysfunction of the disease (Barnett et al., 2006; Stern, 2002, 2003, 2013), it can be hypothesized that the effect may be generalized and thus act also on ToM brain networks.

Furthermore, it is important to underline that our result may also depend on inclusion of current IQ as a CR index. The role of current IQ as an indicator of CR, is a crucial point with pros and cons. Some studies used premorbid IQ, whereas others used WAIS's subtests as indicators of CR (Kim et al., 2015; Nucci et al., 2011; Ferreira et al., 2016).

Last, the influence of CR on dynamic ToM improvement after socio-cognitive trainings, was explored also taking into account the effect of executive functions, as a variable correlated to ToM abilities. We globally observed a significant improvement in ToM performance from baseline, supporting previous results on efficacy of our interventions (Bechi et al., 2015, 2012, 2013), with a significant interaction between CR clusters and time, suggesting an influence of CR profile on treatments outcome. A significant change in ToM measure from baseline to post-treatment evaluation was reported for both Cluster $1(\downarrow \mathrm{PAS} \pm \mathrm{IQ})$ and $2( \pm \mathrm{PAS} \downarrow \mathrm{IQ})$.

Specifically, Cluster $1(\downarrow \mathrm{PAS} \pm \mathrm{IQ})$, characterized by the lowest functioning level but average IQ and intermediate baseline ToM performance, showed a high degree of improvement up to the level that post-treatment scores were comparable to those reached by the "most advantaged" Cluster 3 ( $\uparrow$ PAS $\uparrow I Q$ ). Patients belonging to Cluster 2 ( \pm PAS $\downarrow$ IQ) also obtained a significant improvement, but their ToM performance remained significantly lower than the "most advantaged" Cluster 3 ( $\uparrow$ PAS $\uparrow I Q)$. The latter did not show a significant change after treatment; however, descriptively, we can still observe a trend toward mean higher scores. The absence of significant improvement could depend on a ceiling effect, because Cluster 3 ( $\uparrow$ PAS $\uparrow I Q$ ) was the "best performing" one from the beginning, in terms of ToM functioning.

These results support the role of CR in socio-cognitive domains and further suggest that CR may influence also specific rehabilitation outcome. The importance to define different CR profiles may have both research and clinical relevance. On the one hand, the identification of a precise $\mathrm{CR}$ profile, based on multiple assessments may help to distinguish more homogeneous subgroups of patients, possibly characterized by specific underlying neurophysiological correlates. In line with this, the CR profile may represent a suitable endophenotype for studies aimed at finding factors contributing to the neuroprotective effect exerted by CR. On the other hand, as by definition individuals with high reserve can tolerate more "insults" than those with lower levels (O'Shea et al., 2015), evaluation of CR in clinical setting 
could also have helpful implications. First, it could allow to identify subjects at risk of decline at an earlier stage, giving the opportunity to intervene timely and more intensively with a stronger impact on the course of the disease-associated deterioration.

Moreover, giving the putative predictive effect of $\mathrm{CR}$ on rehabilitation outcome, CR profiles may be used to customize interventions. For instance, our results show that subjects with a lower CR, as assessed by both premorbid functioning and IQ, reach a poorer ToM performance, even after a specific training, suggesting that these patients may benefit from more intensive programs, possibly including preliminary interventions targeting neural plasticity, such as cognitive remediation and brain stimulation techniques. Finally, it is to notice that the rehabilitation treatments, although influenced by the $\mathrm{CR}$ profile, still proved to be overall able to strengthen ToM performance in all patients, regardless of the initial different performance. These data support the hypothesis that experiences at all stages of life, even later ones, can influence CR (Stern, 2013) and that the relationship between CR and rehabilitation may be bidirectional, with also a significant impact of rehabilitative programs on CR.

However, some limitations should be pointed out. First, as already discussed above, the use of current IQ as a CR index, instead of a premorbid measure. Second, the relatively small sample size, that also hampered the use of more factors with possible influence on CR into the cluster analysis. This limit is related with the CR concept (Amoretti et al., 2016; Anaya et al., 2016; de la Serna et al., 2013; Forcada et al., 2015). Third, a longitudinal evaluation is missing and would be relevant to establish the prognostic value of CR. In fact, the nonlongitudinal design of the present study allows to make little inferences regarding the issue of deterioration. This constitutes a crucial factor in CR profiling and has to be taken into account, especially as far as premorbid social and academic functioning is concerned (Cole et al., 2012); by contrast, IQ would tend to be stable after psychosis onset (Leeson et al., 2011).

More specifically, the present study allows only to speculate that Cluster 2 constitutes the group which probably showed a cognitive deterioration, as this is based on the relative discrepancy between earlier functioning and IQ measure and not on PAS trajectory. Furthermore, our findings should be further validated using a premorbid IQ measure, as it constitutes the most reliable indicator of CR. Fourth, the absence in the literature of an assessment able to evaluate the overall ToM construct. In fact, even if PST is one of the more complete tasks evaluating different components of ToM, there are more recent, ecologically valid measures now available to assess ToM, such as TASIT (Bechi et al., 2015; Brüne, 2003b; McDonald, Flanagan, Rollins, \& Kinch, 2003). Moreover, notice that our cluster differentiation and classification were made mainly on the basis of general premorbid functioning, rather than trajectories; this may limit the strength of comparison with previous literature.

In conclusion, this study investigated, in an innovative way, the role of CR in schizophrenia focusing on both ToM abilities and outcome of socio-cognitive treatments. This research is in line with recent literature showing that CR plays a major role all over the lifespan, emphasizing the clinical relevance of defining CR profiles of patients also to customize interventions. A deeper knowledge about $\mathrm{CR}$ may indeed considerably increase our understanding of individual differences and thus improve treatment outcome among patient with schizophrenia.

\section{ACKNOWLEDGMENTS}

Authors Bosia and Cavallaro are joint co-senior authors. The authors declare that they have no conflicts of interest concerning this article. The authors declare that this study had no sponsor.

\section{REFERENCES}

Amoretti, S., Bernardo, M., Bonnin, C.M., Bioque, M., Cabrera, B., Mezquida, G., ... Torrent, C. (2016). The impact of cognitive reserve in the outcome of first-episode psychoses: 2-year followup study. European Neuropsychopharmacology, 26(10), 1638-1648. https://doi.org/10.1016/j.euroneuro.2016.07.003.

Anaya, C., Torrent, C., Caballero, F.F., Vieta, E., Bonnin, C., del, M., ... Ayuso-Mateos, J.L. (2016). Cognitive reserve in bipolar disorder: Relation to cognition, psychosocial functioning and quality of life. Acta Psychiatrica Scandinavica, 133, 386-398. https://doi.org/10.1111/acps.12535.

Anselmetti, S., Poletti, S., Ermoli, E., Bechi, M., Cappa, S., Venneri, A., ... Cavallaro, R. (2008). The brief assessment of cognition in schizophrenia. Normative data for the Italian population. Neurological Sciences, 29(2), 85-92. https://doi.org/ 10.1007/s10072-008-0866-9.

Barnett, J.H., Salmond, C.H., Jones, P.B., \& Sahakian, B.J. (2006). Cognitive reserve in neuropsychiatry. Psychological Medicine, 36(8), 1053-1064. https://doi.org/10.1017/S0033291706007501.

Bechi, M., Bosia, M., Spangaro, M., Buonocore, M., Cocchi, F., Pigoni, A., ... Cavallaro, R. (2015). Combined social cognitive and neurocognitive rehabilitation strategies in schizophrenia: Neuropsychological and psychopathological influences on Theory of Mind improvement. Psychological Medicine, 45(15), 3147-3157. https://doi.org/10.1017/S0033291715001129.

Bechi, M., Riccaboni, R., Ali, S., Fresi, F., Buonocore, M., Bosia, M., ... Cavallaro, R. (2012). Theory of mind and emotion processing training for patients with schizophrenia: Preliminary findings. Psychiatry Research, 198(3), 371-377. https://doi.org/ 10.1016/j.psychres.2012.02.004.

Bechi, M., Spangaro, M., Bosia, M., Zanoletti, A., Fresi, F., Buonocore, M., ... Cavallaro, R. (2013). Theory of Mind intervention for outpatients with schizophrenia. Neuropsychological Rehabilitation, 23(3), 383-400. https://doi.org/ 10.1080/09602011.2012.762751.

Bell, M.D., Johannesen, J.K., Greig, T.C., \& Wexler, B.E. (2010). Memory profiles in schizophrenia: Categorization validity and stability. Schizophrenia Research, 118(1-3), 26-33. https://doi. org/10.1016/j.schres.2009.12.037.

Brenner, H.D., Hodel, B., Roder, V., \& Corrigan, P. (1992). Treatment of cognitive dysfunctions and behavioral deficits in schizophrenia. Schizophrenia Bulletin, 18(1), 21-26.

Brüne, M. (2003a) Social cognition and behaviour in schizophrenia. The Social Brain, 277-313. https://doi.org/10.1002/0470867221. ch13. 
Brüne, M. (2003b) Theory of Mind and the role of IQ in chronic disorganized schizophrenia. Schizophrenia Research, 60(1), 57-64. https://doi.org/10.1016/S0920-9964(02)00162-7.

Cannon-Spoor, H.E., Potkin, S.G., \& Wyatt, R.J. (1982). Measurement of premorbid adjustment in chronic schizophrenia. Schizophrenia Bulletin, 8(3), 470-484.

Cole, V.T., Apud, J.A., Weinberger, D.R., \& Dickinson, D. (2012). Using latent class growth analysis to form trajectories of premorbid adjustment in schizophrenia. Journal of Abnormal Psychology, 121(2), 388-395. https://doi.org/10. 1037/a0026922.

Combs, D.R., Tosheva, A., Wanner, J., \& Basso, M.R. (2006). Remediation of emotion perception deficits in schizophrenia: The use of attentional prompts. Schizophrenia Research, 87(1-3), 340-341. https://doi.org/10.1016/j.schres.2006.05.003.

Cuesta, M.J., Sánchez-Torres, A.M., Cabrera, B., Bioque, M., Merchán-Naranjo, J., \& Corripio, I., . . . PEPs Group. (2015). Premorbid adjustment and clinical correlates of cognitive impairment in first-episode psychosis. The PEPsCog Study. Schizophrenia Research, 164(1-3), 65-73. https://doi.org/ 10.1016/j.schres.2015.02.022.

De la Serna, E., Andrés-Perpiñá, S., Puig, O., Baeza, I., Bombin, I., Bartrés-Faz, D., ... Castro-Fornieles, J. (2013). Cognitive reserve as a predictor of two year neuropsychological performance in early onset first-episode schizophrenia. Schizophrenia Research, 143(1), 125-131. https://doi.org/10.1016/j.schres.2012.10.026.

Dillon, W.R., \& Goldstein, M. (1984). Multivariate analysis: Methods and applications. New York: John Wiley \& Sons Inc.

Evans, J.D., Bond, G.R., Meyer, P.S., Kim, H.W., Lysaker, P.H., Gibson, P.J., \& Tunis, S. (2004). Cognitive and clinical predictors of success in vocational rehabilitation in schizophrenia. Schizophrenia Research, 70(2-3), 331-342. https://doi.org/10.1016/j. schres.2004.01.011.

Ferreira, D., Bartrés-Faz, D., Nygren, L., Rundkvist, L.J., Molina, Y., Machado, A., ... Westman, E. (2016). Different reserve proxies confer overlapping and unique endurance to cortical thinning in healthy middle-aged adults. Behavioural Brain Research, 311, 375-383. https://doi.org/10.1016/j. bbr.2016.05.061.

Fliss, R., Le Gall, D., Etcharry-Bouyx, F., Chauviré, V., Desgranges, B., \& Allain, P. (2016). Theory of Mind and social reserve: Alternative hypothesis of progressive Theory of Mind decay during different stages of Alzheimer's disease. Social Neuroscience, 11(4), 409-423. https://doi.org/10.1080/174709 19.2015.1101014.

Forcada, I., Mur, M., Mora, E., Vieta, E., Bartrés-Faz, D., \& Portella, M.J. (2015). The influence of cognitive reserve on psychosocial and neuropsychological functioning in bipolar disorder. European Neuropsychopharmacology, 25(2), 214-222. https://doi.org/10.1016/j.euroneuro.2014.07.018.

Frith, C.D. (Ed.) (1993). The cognitive neuropsychology of schizophrenia. Hove, UK: Lawrence Erlbaum Associates.

Frith, C.D., \& Corcoran, R. (1996). Exploring 'theory of mind' in people with schizophrenia. Psychological Medicine, 26(3), 521-530.

Frith, U., \& Frith, C.D. (2003). Development and neurophysiology of mentalizing. Philosophical Transactions of the Royal Society of London. Series B, Biological Sciences, 358(1431), 459-473. https://doi.org/10.1098/rstb.2002.1218.

Gilbert, E., Mérette, C., Jomphe, V., Émond, C., Rouleau, N., Bouchard, R.H., ... Maziade, M. (2014). Cluster analysis of cognitive deficits may mark heterogeneity in schizophrenia in terms of outcome and response to treatment. European Archives of Psychiatry and Clinical Neuroscience, 264(4), 333-343. https://doi.org/10.1007/s00406-013-0463-7.

Kay, S.R., Fiszbein, A., \& Opler, L.A. (1987). The positive and negative syndrome scale (PANSS) for schizophrenia. Schizophrenia Bulletin, 13(2), 261-276.

Keefe, R.S.E., Goldberg, T.E., Harvey, P.D., Gold, J.M., Poe, M.P., \& Coughenour, L. (2004). The Brief Assessment of Cognition in Schizophrenia: Reliability, sensitivity, and comparison with a standard neurocognitive battery. Schizophrenia Research, 68(2-3), 283-297. https://doi.org/10.1016/j.schres.2003.09.011.

Kim, S.-G., Lee, E.-H., Hwang, S.-T., Park, K., Chey, J., Hong, S.-H., ... Kim, J.-H. (2015). Estimation of K-WAIS-IV premorbid intelligence in South Korea: Development of the KPIE-IV. The Clinical Neuropsychologist, 29(Supp1), 19-29. https://doi.org/ 10.1080/13854046.2015.1072248.

Kontis, D., Huddy, V., Reeder, C., Landau, S., \& Wykes, T. (2013). Effects of age and cognitive reserve on cognitive remediation therapy outcome in patients with schizophrenia. The American Journal of Geriatric Psychiatry, 21(3), 218-230. https://doi.org/ 10.1016/j.jagp.2012.12.013.

Kurtz, M.M., Gagen, E., Rocha, N.B.F., Machado, S., \& Penn, D.L. (2016). Comprehensive treatments for social cognitive deficits in schizophrenia: A critical review and effect-size analysis of controlled studies. Clinical Psychology Review, 43, 80-89. https://doi.org/10.1016/j.cpr.2015.09.003.

Leeson, V.C., Sharma, P., Harrison, M., Ron, M.A., Barnes, T.R.E., \& Joyce, E.M. (2011). IQ trajectory, cognitive reserve, and clinical outcome following a first episode of psychosis: A 3-year longitudinal study. Schizophrenia Bulletin, 37(4), 768-777. https://doi.org/10.1093/schbul/sbp143.

Levitt, J.J., O’Donnell, B.F., McCarley, R.W., Nestor, P.G., \& Shenton, M.E. (1996). Correlations of premorbid adjustment in schizophrenia with auditory event-related potential and neuropsychological abnormalities. The American Journal of Psychiatry, 153(10), 1347-1349. https://doi.org/10.1176/ajp.153.10.1347.

Lysaker, P.H., Salvatore, G., Grant, M.L.A., Procacci, M., Olesek, K.L., Buck, K.D., ... Dimaggio, G. (2010). Deficits in theory of mind and social anxiety as independent paths to paranoid features in schizophrenia. Schizophrenia Research, 124(1-3), 81-85. https://doi.org/10.1016/j.schres.2010.06.019.

Mazza, M., De Risio, A., Surian, L., Roncone, R., \& Casacchia, M. (2001). Selective impairments of theory of mind in people with schizophrenia. Schizophrenia Research, 47(2-3), 299-308.

McDonald, S., Flanagan, S., Rollins, J., \& Kinch, J. (2003). TASIT: A new clinical tool for assessing social perception after traumatic brain injury. The Journal of Head Trauma Rehabilitation, 18(3), 219-238. https://doi.org/http://dx.doi.org/10.1097/00001199200305000-00001.

McGurk, S.R., Moriarty, P.J., Harvey, P.D., Parrella, M., White, L., Friedman, J., \& Davis, K.L. (2000). Relationship of cognitive functioning, adaptive life skills, and negative symptom severity in poor-outcome geriatric schizophrenia patients. The Journal of Neuropsychiatry and Clinical Neurosciences, 12(2), 257-264. https://doi.org/10.1176/jnp.12.2.257.

Neumann, C.S., Baum, K.M., Walker, E.F., \& Lewine, R.L. (1996). Childhood behavioral precursors of adult neuropsychological fuctioning in schizophrenia. Neuropsychiatry, Neuropsychology, and Behavioral Neurology, 9, 221-229.

Nucci, M., Mapelli, D., \& Mondini, S. (2011). Cognitive Reserve Index questionnaire (CRIq): A new instrument for measuring cognitive reserve. Aging Clinical and Experimental Research, 24(3), 218-226. https://doi.org/10.3275/7800. 
O'Shea, D.M., Fieo, R.A., Hamilton, J.L., Zahodne, L.B., Manly, J.J., \& Stern, Y. (2015). Examining the association between late-life depressive symptoms, cognitive function, and brain volumes in the context of cognitive reserve. International Journal of Geriatric Psychiatry, 30(6), 614-622. https://doi.org/10.1002/gps.4192.

Ochoa, S., Huerta-Ramos, E., Barajas, A., Iniesta, R., Dolz, M., Banos, I., ... Usall, J. (2013). Cognitive profiles of three clusters of patients with a first-episode psychosis. Schizophrenia Reseaarch, 150(1), 151-156. https://doi.org/10.1016/j.schres.2013.07.054.

Penn, D.L., \& Combs, D. (2000). Modification of affect perception deficits in schizophrenia. Schizophrenia Research, 46(2-3), 217-229.

Pickup, G.J., \& Frith, C.D. (2001). Theory of mind impairments in schizophrenia: Symptomatology, severity and specificity. Psychological Medicine, 31(2), 207-220.

Renwick, L., Lyne, J., Donoghue, B.O., Owens, L., Doyle, R., Hill, M., ... Clarke, M. (2015). Prodromal symptoms and remission following first episode psychosis. Schizophrenia Research, 168(1-2), 30-36. https://doi.org/10.1016/j.schres.2015.07.001.

Roder, V., Brenner, H.D., Müller, D., Lächler, M., Zorn, P., Reisch, T., ... Schwemmer, V. (2002). Development of specific social skills training programmes for schizophrenia patients: Results of a multicentre study. Acta Psychiatrica Scandinavica, 105(5), 363-371. https://doi.org/10.1034/j.1600-0447.2002.10214.x.

Steiger, J.H. (2004). Beyond the F test: Effect size confidence intervals and tests of close fit in the analysis of variance and contrast analysis. Psychological Methods, 9(2), 164-182. https:// doi.org/10.1037/1082-989X.9.2.164.

Stern, Y. (2002). What is cognitive reserve? Theory and research application of the reserve concept. Journal of the International Neuropsychological Society, 8(3), 448-460.

Stern, Y. (2003). The concept of cognitive reserve: A catalyst for research. Journal of Clinical and Experimental Neuropsychology, 25(5), 589-593. https://doi.org/10.1076/jcen.25.5.589.14571.

Stern, Y. (2013). Cognitive reserve: Implications for assessment and intervention. Folia Phoniatrica et Logopaedica, 65(2), 49-54. https://doi.org/10.1159/000353443.

Velligan, D.I., Mahurin, R.K., Diamond, P.L., Hazleton, B.C., Eckert, S.L., \& Miller, A.L. (1997). The functional significance of symptomatology and cognitive function in schizophrenia. Schizophrenia Research, 25(1), 21-31. https://doi.org/10.1016/S09209964(97)00010-8.

Wechsler, D. (1997). Wechsler Adult Intelligence Scale - Revised. New York: The Psychological Corp. 